\title{
Observations of Internal Charging Currents in Medium Earth Orbit
}

\author{
Keith A. Ryden, Member, IEEE, Paul A. Morris, Karen A. Ford, Alexander D. P. Hands, \\ Clive S. Dyer, Senior Member, IEEE, Ben Taylor, Member, IEEE, Craig I. Underwood, Member, IEEE, \\ David J. Rodgers, Giuseppe Mandorlo, Giuliano Gatti, Hugh D. R. Evans, and Eamonn J. Daly
}

\begin{abstract}
The Galileo global navigation system will employ an array of satellites in medium Earth orbit (MEO). Internal charging is one of the primary hazards for any spacecraft in MEO, and accordingly, the Galileo test satellite, Giove-A, carries a detector, SURF, to undertake the measurement of internal charging currents at three different shielding depths. The currents are due to electrons stopped in three aluminum plates: the first plate is $0.5 \mathrm{~mm}$ thick and located under $0.5-\mathrm{mm}$ Al-equivalent shielding, the second is $0.5 \mathrm{~mm}$ thick and located underneath the first plate, and the third is $1.0 \mathrm{~mm}$ thick and located underneath the second plate. Giove-A was successfully launched on December 28, 2005 into a $23300-\mathrm{km}$ circular $56^{\circ}$ inclination orbit. In this paper, we provide data on the internal charging currents observed in 2006, with particular emphasis on two large charging events, one in mid-April and one in mid-December. Comparisons are made to predictions using the DICTAT internal charging tool and the FLUMIC "worst case" trapped electron belt model. In general, the charging currents observed are safely within the standard DICTAT 3.5/FLUMIC 3.0 predictions but are exceeded in the most shielded plate on five calendar days during December 2006. The December event was unique in 2006 in that it was triggered by the arrival of a coronal mass ejection (CME) rather than being due to the effects of a fast solar wind stream flowing from a coronal hole. The currents measured in the December event were, however, an order of magnitude lower than those predicted using the "anomalously large event" supplement to FLUMIC which models the most extreme electron enhancements associated with CMEs.
\end{abstract}

Index Terms-Energetic electron belts, internal charging, medium Earth orbit (MEO).

\section{INTRODUCTION}

$\mathbf{T}$ HE EUROPEAN Galileo navigation system will employ a constellation of 30 satellites in circular medium Earth orbit (MEO) of altitude of approximately $23300 \mathrm{~km}$ and inclination of $\sim 56^{\circ}$. Each satellite will pass close to the heart of

Manuscript received January 30, 2008; revised April 8, 2008 and June 16, 2008. Current version published November 14, 2008. This work was supported in part by the European Space Agency, by Surrey Satellite Technology Ltd., and by the QinetiQ Fellowship scheme.

K. A. Ryden, P. A. Morris, K. A. Ford, A. D. P. Hands, and C. S. Dyer are with QinetiQ, Farnborough, GU14 0LX Hampshire, U.K. (e-mail: karyden@qinetiq.com; pamorris@qinetiq.com; kaford@qinetiq.com; ahands@qinetiq.com; csdyer@qinetiq.com).

B. Taylor and C. I. Underwood are with the University of Surrey, GU2 7XH Guildford, U.K. (e-mail: b.taylor@ surrey.ac.uk).

D. J. Rodgers, G. Mandorlo, G. Gatti, H. D. R. Evans, and E. J. Daly are with the European Space Agency, 2200 AG Noordwijk, The Netherlands (e-mail: david.rodgers@esa.int).

Color versions of one or more of the figures in this paper are available online at http://ieeexplore.ieee.org.

Digital Object Identifier 10.1109/TPS.2008.2001945 the Earth's outer trapped radiation belt twice in each 14-h orbit, and thus, energetic electrons will form a significant component of the operational environment. It is well known that the outer belt is highly dynamic and prone to regular enhancements of the energetic electron population which can be triggered by persistent fast solar wind streams or coronal mass ejections (CMEs) [1], [2]. Many anomalies on Earth-orbiting satellites have been correlated with such electron belt enhancements [3], [4], and these anomalies are frequently attributed to the process of internal charging [5]-[7]. In this mechanism, energetic electrons penetrate the outer shielding of the spacecraft but are eventually brought to a stop, therefore depositing a charge at the end of their track. During enhancement events, the flux of penetrating electrons can increase by orders of magnitude. If, at a particular location, the charge leakage rate is low compared to the arrival rate (e.g., dielectrics or isolated metal items), then over time (typically one to two days), enough charge can be deposited for dielectric strengths to be exceeded and an internal electrostatic discharge may occur. Such discharges can cause an electronic circuit upset or even permanent damage to an electronic component. This process has been reproduced in both flight- and ground-based experiments, e.g., [8]-[10]. Hence, there is strong interest in monitoring charging rates at typical shielding depths in the Galileo orbit so that protection strategies can be optimized.

The European Space Agency (ESA) has launched a test satellite, Giove-A, into a $23300-\mathrm{km}$ circular $56^{\circ}$ inclination orbit. One of its objectives is to measure the radiation environment encountered in MEO. Some initial data from the two monitors onboard, "Merlin" and "CEDEX," have already been reported [11]. Merlin, shown in Fig. 1, provides monitoring of internal charging rates, total ionizing doses, energetic proton fluxes, and ion linear energy transfer spectra. Of particular interest for charging studies is the "SURF" detector located within Merlin, which measures internal charging currents at three different shielding depths.

\section{SURF DETECTOR Within MERLiN}

The SURF detector was first described in 1999 [12] and was proven in space in 2000 on the STRV1d satellite in geostationary transfer orbit [13]. For the Giove-A mission, SURF comprises three shielded aluminum collector plates mounted in a stack as shown in Fig. 2. The first plate is $0.5 \mathrm{~mm}$ thick and located under a 0.5 -mm Al-equivalent shielding; the second is $0.5 \mathrm{~mm}$ thick and located under the first plate (plus shield); the 


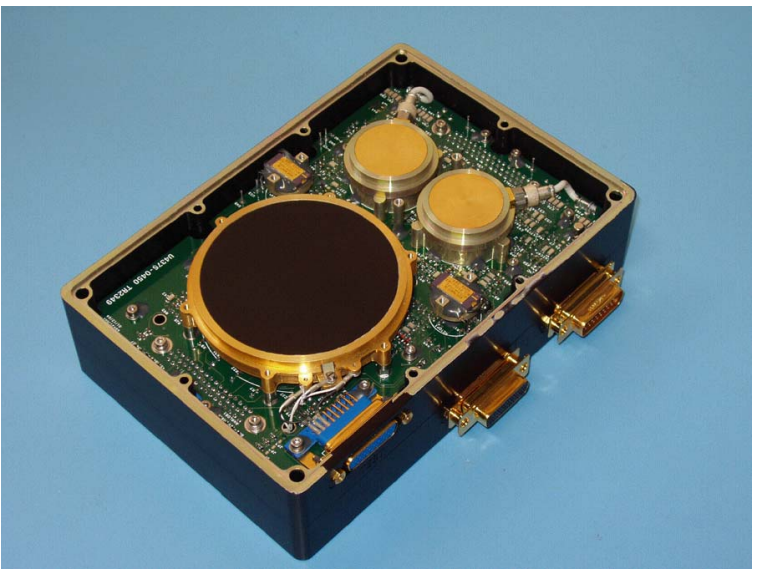

Fig. 1. Merlin-Giove-A instrument showing the main detector board: SURF is the large diameter circular detector. Also visible are the particle telescopes for protons and ions and the RadFETs for total dose measurement. The power and data handling functions are located on the lower board (hidden).

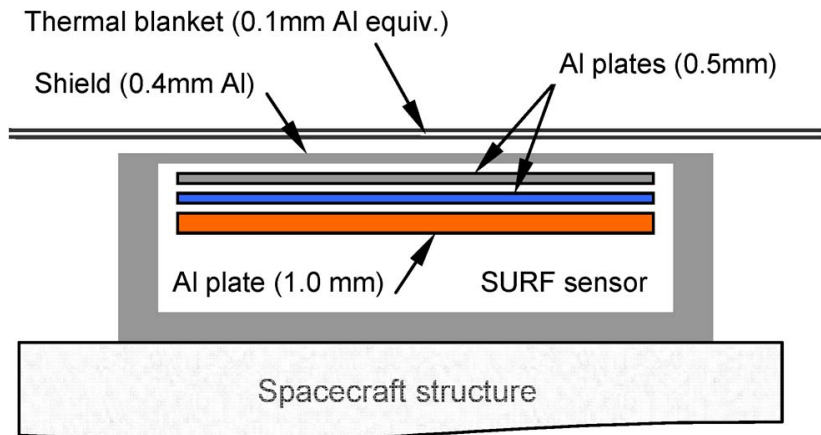

Fig. 2. General configuration of the SURF shielded plates in Merlin-Giove-A.

third is $1.0 \mathrm{~mm}$ thick and located under the second plate. Each of the three collector plates is connected to an electrometer to measure deposited current. These dimensions are representative of typical thicknesses of dielectrics within spacecraft. The plate diameter $(70 \mathrm{~mm})$ is chosen to ensure that currents are within a measurable range when within the belt, allowing sufficient headroom to ensure that exceptionally severe environments will be captured. The dominant particle type is indicated by the polarity of the current detected. Within the electron belts, we expect (and observe) this current to be negative, i.e., the deposition of electrons in the plates is always dominant. Any deposited protons will of course contribute a positive current but the outer belt proton population is negligible except during solar particle events (SPEs). Of course, from a charging perspective, it is only the net current which is of importance; therefore, SURF accurately reports the charging threat. Simulations using MULASSIS (see Appendix A) show that, even during major SPEs, the magnitude of the proton currents are small compared to the electron currents. In fact, this resilience to the effects of protons, confirmed by earlier observations from within the intense inner belt [9], means that SURF is also useful as an electron detector in its own right because traditional devices (based on particle counting) are often readily contaminated in mixed electron/proton environments.

SURF was calibrated prior to launch by injecting known currents into the plates: the calibration is traceable to national

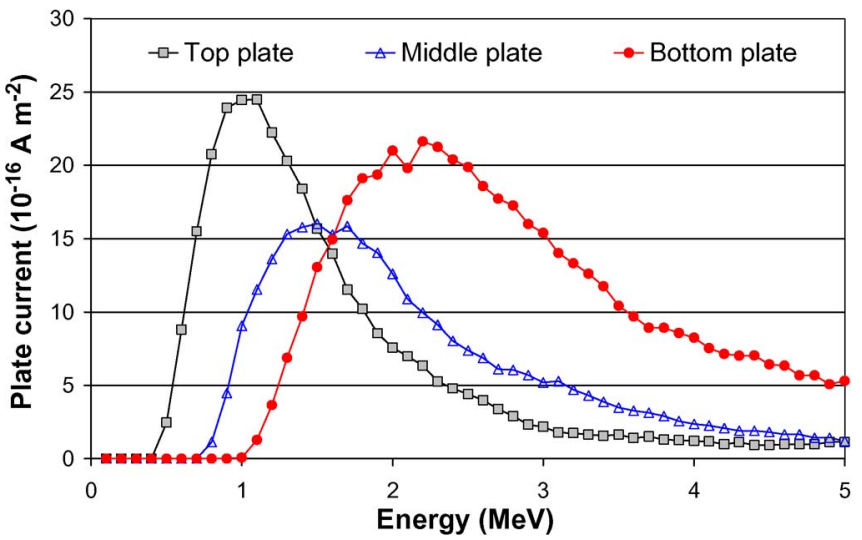

Fig. 3. Response functions for the three SURF plates obtained using the DICTAT tool. The environment was isotropic with intensity of $1 \mathrm{e} \mathrm{cm}^{-2} \mathrm{sr}^{-1} \mathrm{~s}^{-1}$.

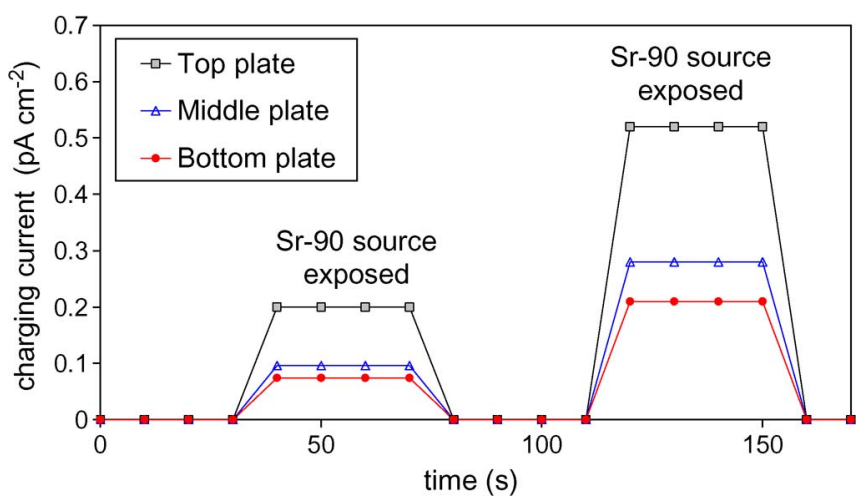

Fig. 4. Currents detected in the three SURF plates during preflight test exposures in the REEF. The results here correspond to two different ranges of the electron point source (Sr-90 pellet) from the top plane of the Merlin unit.

standards. Because the detector itself is inert (i.e., metal plate), the amplifier chain is the only potential cause of calibration drift. To monitor any variation in electrometer performance, a control channel is provided which is simply an identical electrometer/amplifier chain not connected to any plate. The control channel enables any drift to be corrected, although this has not been needed to date.

Simple 1-D modeling of the SURF plates using the ESA DICTAT tool [14], [15] has enabled the approximate response functions to be plotted-see Fig. 3. The electron environment selected within DICTAT at each discrete energy point was isotropic with intensity of $1 \mathrm{e} \mathrm{cm}^{-2} \mathrm{sr}^{-1} \mathrm{~s}^{-1}$. According to these results the electron energies of peak sensitivity for each plate are approximately 1.0, 1.5, and $2.1 \mathrm{MeV}$ for top, middle, and lower plates, respectively.

Prior to launch, SURF was tested in the QinetiQ Realistic Electron Environment Facility (REEF) which simulates the outer belt electron environment using a 3.7-GBq strontium90 beta emitter [16]. Sr-90 produces a continuous spectrum of electrons from 0 up to $2.2 \mathrm{MeV}$. The results from two in vacuum exposures with different source-to-target ranges are shown in Fig. 4. These exposures provided confirmation of preflight operation and also enable cross-checking of model results against a reference case. 


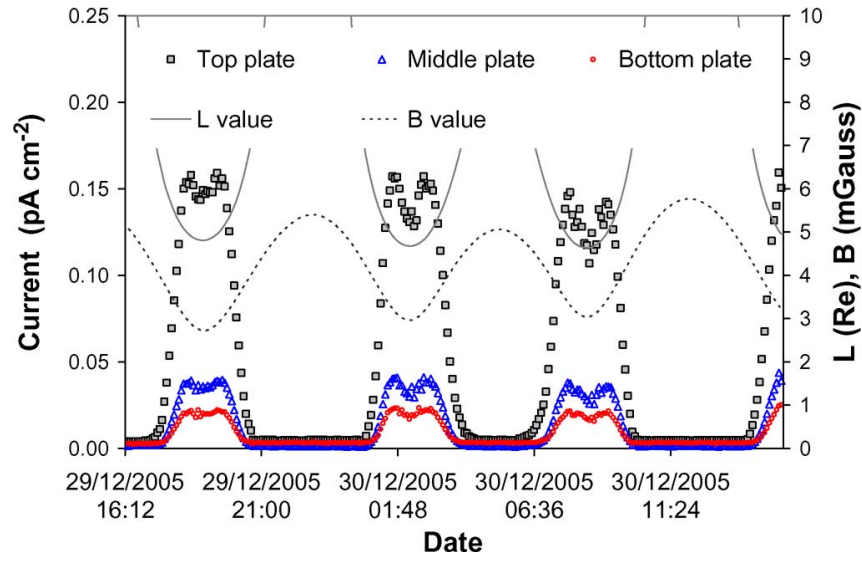

Fig. 5. Magnitude of the (negative) internal charging currents measured by SURF in the $24 \mathrm{~h}$ after unit switch-on. Also plotted are geomagnetic coordinates $B$ and $L$. Each passage through the outer belt is clearly seen with a notable peak on either side of the crossing of the geomagnetic equator (minima in $B$ ). The small currents registered outside of the belt (large $L$ ) are instrument bias currents.

\section{Flight DATA}

SURF has been operational on Giove-A since shortly after the launch on 28 December 2005. Fig. 5 is a plot of the magnitude of the currents (picoamperes per square centimeter) detected in the three plates for the first $24 \mathrm{~h}$ after SURF activation. All currents observed are negative in polarity. Also shown in Fig. 5 are the $B, L$ values obtained from the Tsyganenko 89 [17] external magnetic field model. The internal field model was IGRF/DGRF with epoch set at 1994. Each passage of Giove-A through the outer belt is evident along with a distinctive "double peak"- this feature arises due to the circular orbital path of Giove-A through the outer belt, which possesses a crescent-shape cross section. On these days, Giove-A crosses the geomagnetic equatorial plane below the $L$-value where the flux is at its peak.

It is interesting to compare the relative magnitude of the currents in the three plates to those seen in the REEF tests. While following the same order, i.e., $J_{\text {top }}>J_{\text {middle }}>J_{\text {bottom }}$ where $J$ is the current density deposited in each plate; the ratios between the currents are greater in the flight data from late December 2005 indicating a softer electron spectrum at that time than that provided by REEF.

Fig. 6 is a plot of the SURF internal charging currents measured at the crossings of the geomagnetic equator $\left(B / B_{0} \sim 1\right)$ from January-June 2006. These crossings correspond to $L$ values of approximately 4.75 in quiet times. Giove-A was launched at the beginning of a period of outer belt enhancement, which peaked a few days later and then subsided over the next few weeks. A new belt enhancement occurred in late January 2006 and enhancement events continued to occur at approximately 27-day (i.e., solar rotation) intervals throughout the first six months of 2006. These enhancements were associated with a persistent midlatitude solar coronal hole, first observed in October 2005 as STAR catalog no. CH 192 [18], which sent fast $\left(>700 \mathrm{~km} \mathrm{~s}^{-1}\right)$ solar wind streams toward the Earth at approximately 27-day intervals (synodic solar rotation period). This particular coronal hole was very long lived, lasting through to September 2006. The large event in mid-April is

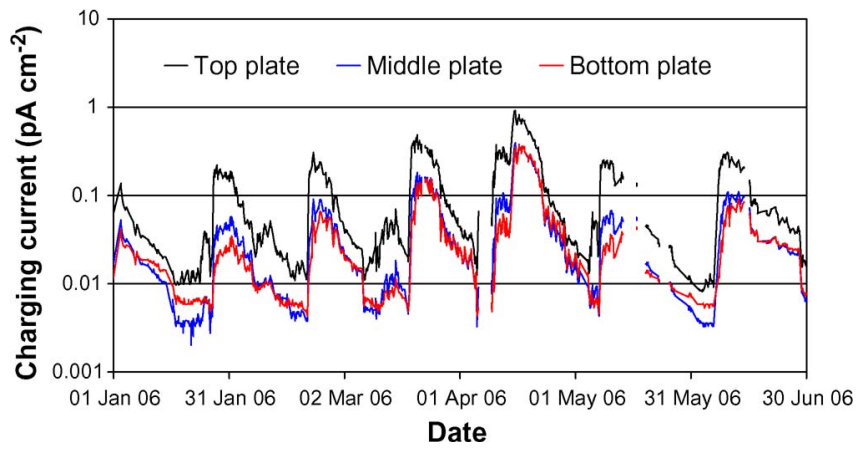

Fig. 6. Internal charging currents detected at the crossings of the geomagnetic equator (i.e., $B / B_{0} \sim 1$ ) from January to June 2006 by the SURF detector on Giove-A. The points plotted correspond to $L \sim 4.75$. Electron belt enhancements occur roughly every 27 -day solar rotation interval over this period.

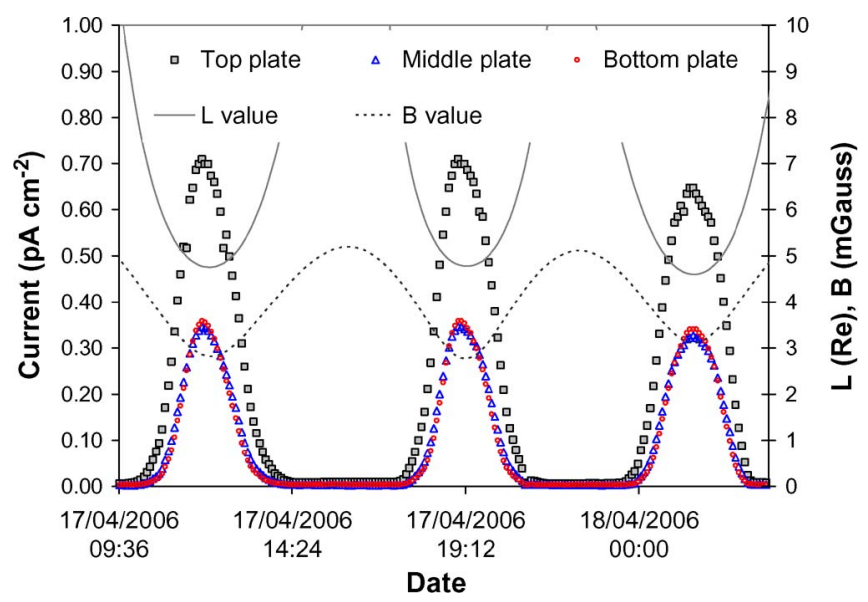

Fig. 7. Profile of charging currents around the orbit during the electron enhancement event in April 2006. A single peak is now observed together with a harder spectrum. Note that the current in the bottom plate is very similar to that in the middle plate, noting of course that the bottom plate is thicker than the other two.

associated with the improved efficiency of electron acceleration at the equinoxes.

During the April event, the profile of the currents around the orbit was significantly modified as shown in Fig. 7. The double peak has disappeared which suggests that the location of the peak of the outer belt in the plane of the geomagnetic equator has moved to a lower $L$-value, i.e., below that at which Giove-A crosses the geomagnetic equator. The charging currents shown in Fig. 7 now indicate a harder electron spectrum than that provided by REEF. The ratio $J_{\mathrm{top}} / J_{\text {middle }}$ at the geomagnetic equator crossing had a value of approximately two in mid-April 2006, whereas it was as high as four in late December 2005. The ratio $J_{\text {middle }} / J_{\text {bottom }}$ was about one in mid-April 2006 but about two in late December 2005. While the $J_{\text {middle }} / J_{\text {bottom }}$ ratio of $\sim 1$ may at first seem surprising, it should be recalled that the bottom plate is twice as thick as the middle plate.

Fig. 8 shows internal charging currents at the crossings of the geomagnetic equator $\left(B / B_{0} \sim 1\right)$ for the second half of 2006. Enhancements continued to occur frequently over this period, although the picture was more complex due to the appearance and decay of a number of midlatitude coronal holes. In addition, an Earth-directed CME occurred on December 13, 


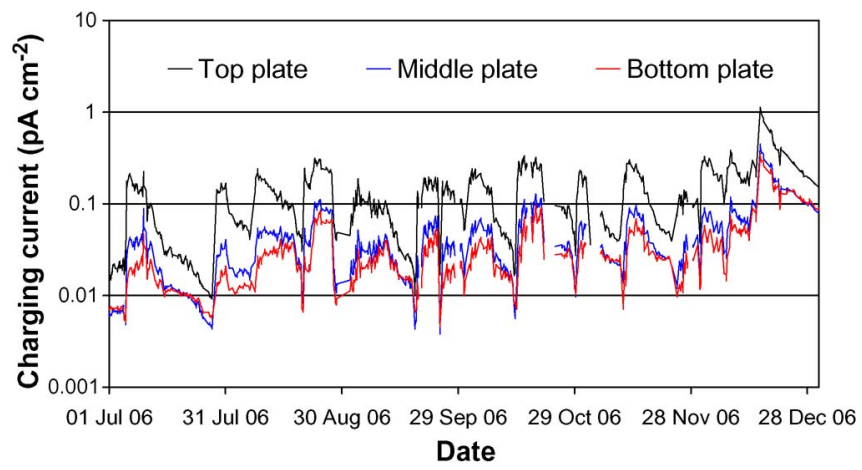

Fig. 8. Internal charging currents recorded at the crossings of the geomagnetic equator (i.e., $B / B_{0} \sim 1$ ) in MEO from July to December 2006 by the SURF detector. The points plotted correspond to $L \sim 4.75$.

2006 [19], [20] which led to an outer belt enhancement of similar magnitude to that seen in April 2006.

\section{COMPARISON TO THE DICTAT/FLUMIC ENGINEERING MODEL}

Electron enhancement events are of engineering significance, and we need to compare the observed data to worst case models used when designing protection for satellites. DICTAT is ESA's engineering tool for evaluation of internal charging hazards [14] and is available worldwide via the Spenvis system [15]. DICTAT models the electron environment around the orbit, determines the electron transport and deposition rates, and also calculates the electric fields within dielectrics. DICTAT is particularly valuable in that it can cope with any Earth orbit including MEO.

For the outer belt electron environment, DICTAT uses the FLUMIC model [21] which is an empirical worst case flux model specifically created for the purpose of internal charging analysis and protection. The FLUMIC model creates a worst case envelope around the peak one-day fluxes recorded by various detectors over the 1980s and 1990s and generalizes to other regions and periods. The model returns one-day mean fluxes because this is the time period relevant to internal charging in typical dielectrics-instantaneous peaks are not of great significance. The creators of FLUMIC necessarily made many assumptions and simplifications due to the paucity of measurements available, the variety of detectors from which data are drawn, uncertainties over calibration, very limited sampling of energy, $L$ and $B$, and, in many cases, the sporadic nature of coverage periods. FLUMIC gives the one-day mean electron flux as a function of the parameters $B, L$, season of the year, and fraction of the solar cycle. The version of FLUMIC referred to in this paper is 3.0 [22].

For electron transport and deposition, DICTAT uses an analytical approach in line with the need for rapid run times. In addition, the scale of uncertainties in both the environment and material parameters would not justify the attempt to achieve a very high level of accuracy in the radiation transport aspects of the code. The version of DICTAT referred to in this paper is 3.5.

Data from SURF are a valuable test of the output from the first two stages of DICTAT, i.e., the electron environment
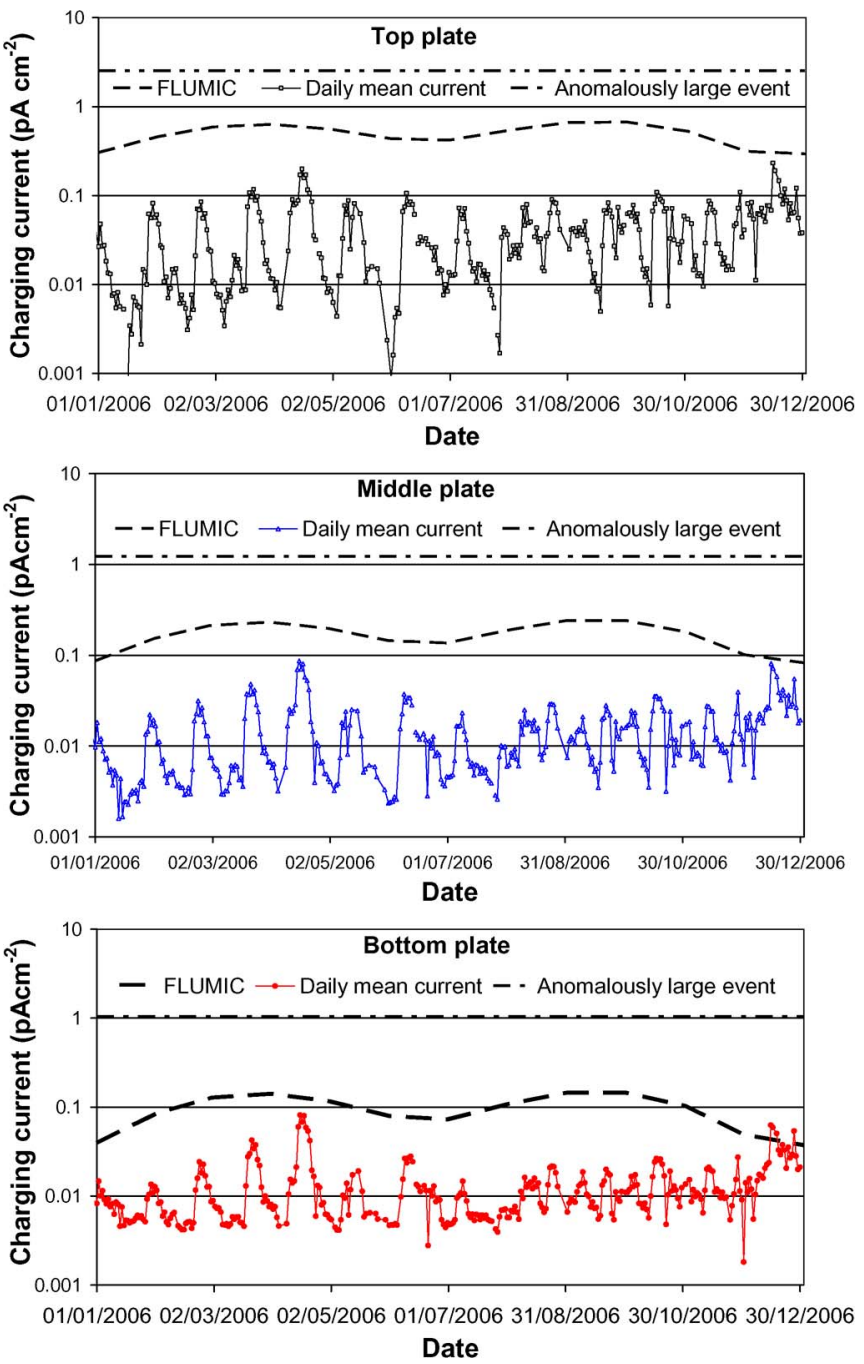

Fig. 9. One-day averages of internal charging current in the SURF plates compared to the DICTAT 3.5 predictions. The DICTAT predictions using both FLUMIC 3.0 and ALE electron environments are shown. The standard DICTAT/FLUMIC prediction is breached in the bottom plate during the December event.

and the electron transport/deposition calculations. The DICTAT 3.5/FLUMIC 3.0 model has been run to calculate the predicted internal charging currents in the collector plates for the Giove-A orbit over 2006. The one-day mean currents predicted by DICTAT/FLUMIC are compared to the Giove-A SURF data from 2006 in Fig. 9. A one-day mean of SURF data is plotted because this is the timescale on which the FLUMIC model is based. It can be seen that, in general, a margin of safety exists between predictions and observations as would be expected for a "worst-case" model such as FLUMIC.

The peak currents observed in each plate are quite similar in the April and December events (the peak days for the top plate were April 15 and December 15, respectively). Whereas the DICTAT/FLUMIC envelope is not exceeded in April, it is exceeded for the bottom plate on five separate days in December. This is because the seasonal modulation function within FLUMIC depresses the predicted currents near the solstices. The currents observed in December are however still within the DICTAT predictions for the equinoxes. 
As mentioned above, the December enhancement was associated with a CME rather than a fast solar wind stream from a coronal hole. In fact, CMEs can produce some of the most severe outer belt enhancements, and the FLUMIC model includes a supplement to cover these, albeit rare, "anomalously large enhancements" (ALEs). The currents predicted using the ALE spectrum as an input to DICTAT are also shown in Fig. 9 on each panel. Note that the ALE supplement has no seasonal or solar cycle dependence. The observed currents in the December CME-induced enhancement are less than those predicted using the ALE spectrum by about an order of magnitude. Further occurrences of SPE-/CME-related enhancements are expected as we move toward solar maximum.

\section{EXAMINATION OF SPECTRA}

FLUMIC models the worst case electron energy spectrum using a simple exponential function together with an associated e-folding energy

$$
F(>E)=F_{0} \cdot \exp -\left(E / E_{0}\right)
$$

where

$$
\begin{array}{ll}
E & \text { electron energy }(\mathrm{MeV}) ; \\
E_{0} & \text { folding energy }(\mathrm{MeV}) ; \\
F(>E) & \text { integral flux (per square centimeter per second per } \\
& \text { steradian); } \\
F_{0} & \text { integral flux } E>0 \text { (per square centimeter per } \\
& \text { second per steradian). }
\end{array}
$$

Note that the validity of FLUMIC is limited to E $>200 \mathrm{keV}$ because it does not attempt or need to model the low energy electron population.

With knowledge of the plate response functions (see Section II), it is possible to derive a best fit exponential spectrum (i.e., $E_{0}, F_{0}$ ) from the SURF data for any point in time. A simple method has been used to carry out this task as described in Appendix B. For the internal charging problem, the spectrum during periods when fluxes are at their highest is of greatest concern. The fitted spectra derived from SURF data for the peak days (in terms of current in the top plate) of the April and December enhancements (i.e., fifteenth of each month) are shown in Fig. $10\left(L \sim 4.75, B / B_{0} \sim 1\right)$ along with the FLUMIC 3.0 spectra for the same dates. Also, shown is the ALE spectrum and the AE8 min spectrum [23] for $L=4.75$, $B / B_{0}=1$. The observed spectra for the two peak days are quite similar, although harder than predicted by the standard FLUMIC. The gradients of the lines (i.e., "hardness") are in fact closer to the ALE slope than to those obtained from the standard FLUMIC model.

In practice, the spectrum changes continually. The value of $E_{o}$ (obtained from the fitting process) at each geomagnetic equator crossing throughout 2006 is shown in Fig. 11 along with the $>0.6$ - and $>2-\mathrm{MeV}$ fluxes (similarly obtained). Repeating cycles of hardening and softening of the electron spectrum are evident, and these are well correlated with the electron enhancements. The spectrum hardens as each event progresses and then softens again suddenly at the start of the next.

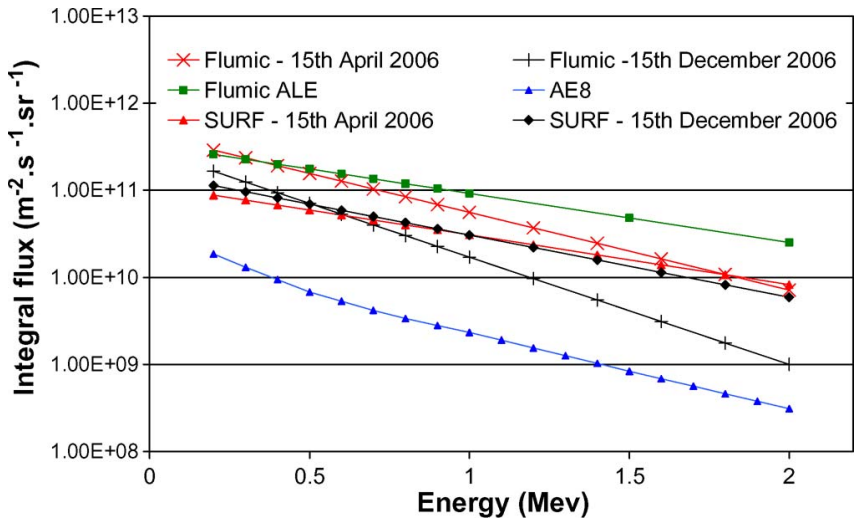

Fig. 10. Observed electron spectra for April 15, 2006 and December 15, 2006 ( $\left.L=4.75, B / B_{0}=1\right)$ compared with the FLUMIC (v3.0), ALE, and AE8 min spectra for the same location. Of course, AE8 is an average model rather than a worst case and is provided for reference only.

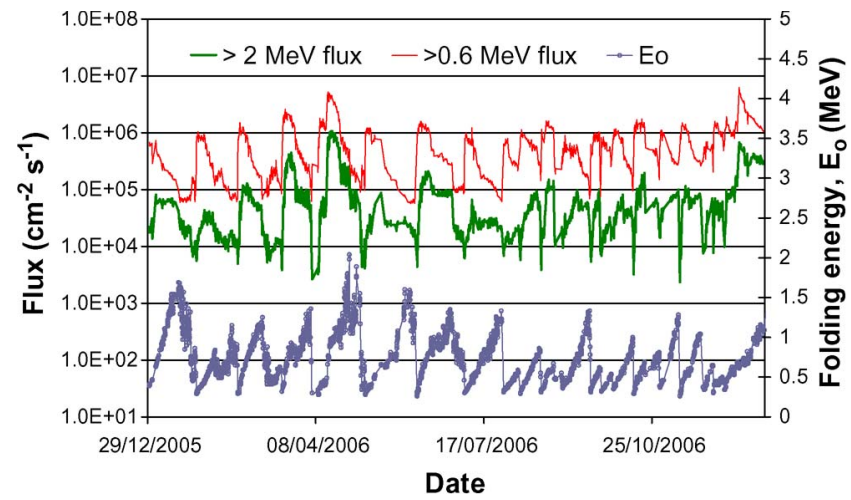

Fig. 11. Electron fluxes and e-folding energies at the Giove-A geomagnetic equator crossings in 2006. This plot is obtained from the fitting of simple exponential spectra to the SURF internal charging data. Repeated hardening and softening of the spectrum is well correlated with the enhancement events. Note that a larger $E_{o}$ indicates a harder spectrum.

Closer examination of Fig. 11 shows that, in the December enhancement event, the peak fluxes of both $>0.6$ - and $>2-\mathrm{MeV}$ electrons occurred on the same day (i.e., December 15) and $E_{o}$ was $0.61 \mathrm{MeV}$. In the April event, the peak flux of $>0.6-\mathrm{MeV}$ electrons occurred two days prior to the peak flux of $>2-\mathrm{MeV}$ electrons which occurred on April 17. In this case, $E_{o}$ was $0.76 \mathrm{MeV}$ on April 15, increasing to $0.96 \mathrm{MeV}$ on the seventeenth.

\section{Comparison to GOES Measurements}

The NOAA GOES satellites provide continuous monitoring of the outer electron belt [24] from geostationary orbit: the GOES > 2.0- and $>0.6-\mathrm{MeV}$ electron flux measurements are widely used for internal charging studies. A comparison with the SURF MEO data is thus of interest even though the $B$, $L$ ranges of the two missions do not actually overlap. Fig. 12 shows a plot comparing the $>2-\mathrm{MeV}$ flux reported by GOES12 with the $>2-\mathrm{MeV}$ flux derived from fitting the SURF data to exponential form. From this plot, it appears that many enhancement events are more persistent in MEO than GEO. 


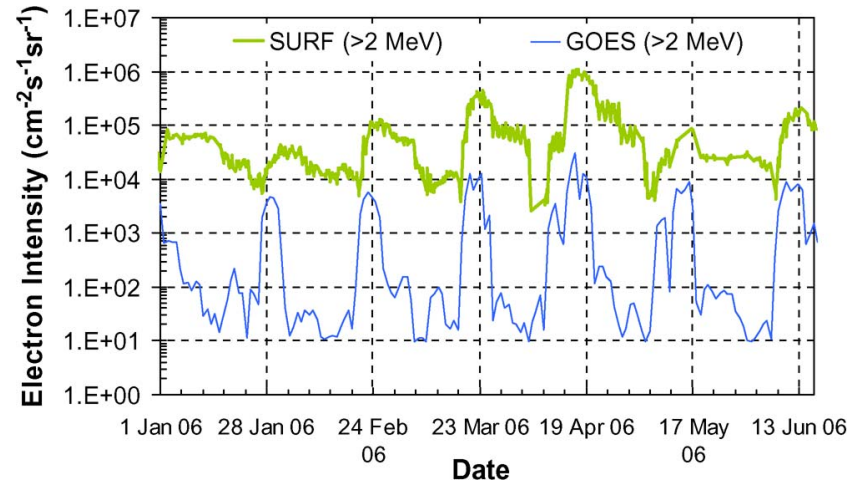

Fig. 12. Comparison of the GOES-12 daily mean $>2-\mathrm{MeV}$ (geostationary orbit) and SURF $>2-\mathrm{MeV}$ fluxes (geomagnetic equator crossings) for the first half of 2006. Also, plotted are 27.2-day gridlines (i.e., synodic rotation period of the Sun for midlatitudes).

\section{CONCLUSION}

The SURF detector within Merlin has recorded internal charging currents at three shielding depths in MEO throughout 2006. These charging data show in particular the effects of recurrent outer belt enhancements due to the influence of midlatitude solar coronal holes. The enhancements display a very clear periodicity of approximately 27 days in the first half of the year. Regular enhancements are in line with expectations for this part of the solar cycle (close to solar minimum) because coronal holes continue to occur frequently, even though the peak period is usually in the declining phase of the solar cycle. The internal charging currents detected around the Giove-A orbit are seen to vary considerably in profile both within and between enhancement events.

There were two enhancements in 2006 which were notably larger than the rest, one in mid-April and one in mid-December. The former was associated with a fast solar wind stream coupled with the efficient energization of the belt near an equinox, whereas the latter was associated with the arrival of a CME. The peak instantaneous currents recorded during these two events were quite similar, being approximately $1 \mathrm{pA} \mathrm{cm}{ }^{-2}$ for the top plate $(0.5-\mathrm{mm} \mathrm{Al}$ shield, $0.5-\mathrm{mm} \mathrm{Al}$ collector) and $0.4 \mathrm{pA} \mathrm{cm}^{-2}$ for both the middle plate $(1.0-\mathrm{mm} \mathrm{Al}$ shield, $0.5-\mathrm{mm} \mathrm{Al}$ collector) and the bottom plate $(1.5-\mathrm{mm} \mathrm{Al}$ shield, 1.0-mm Al collector). The observed one-day mean charging currents in the three SURF plates have remained within the FLUMIC/DICTAT worst case envelope over the year except for five calendar days during December when the current in the bottom plate exceeded the prediction (although still within the DICTAT/FLUMIC worst case for 2006 which occurs at the equinoxes). An improvement to DICTAT would be for the user to input simply the start and end dates of the mission to ensure that the seasonal modulation is fully accounted for, rather than having to choose a specific date to run the model.

As mentioned before, the December "large" event was associated with a CME, and FLUMIC contains a supplementary "ALE" model to estimate worst case fluxes for such events. The one-day SURF currents observed in December 2006 were an order of magnitude below those estimated when using the FLUMIC ALE spectrum, i.e., the December event represented only a modest enhancement. Much larger enhancements can be anticipated closer to solar maximum.

It is worth noting that some CME-related electron enhancements are associated with SPEs which can render counter-type electron detectors unusable. SURF-type detectors would then be invaluable as an electron monitors due to their resilience to proton contamination.

The relative currents in the three plates give information on spectral hardness which indicates a general trend for the hardening of the electron spectrum during the course of the enhancements. This is followed by a sudden softening at the start of the next event. On the peak days of the April event, the folding energies of the spectra were found to lie between 0.76 and $0.96 \mathrm{MeV}$, somewhat higher than the $0.49 \mathrm{MeV}$ provided by the standard FLUMIC. The value of $E_{o}$ on December 15 was $0.61 \mathrm{MeV}$, a little lower than the $0.77 \mathrm{MeV}$ provided by the ALE supplement to FLUMIC which aims to model this type of CME-related event.

Of course, the period covered by our SURF measurements in MEO represents, as yet, only a small part of the solar cycle. Even so, these data provide a useful test of the available environment models and design tools. In due course, further data will enable updates of DICTAT, FLUMIC, and other tools to be accomplished. Data on doses, ions, and proton fluxes will be examined elsewhere.

\section{APPENDIX A \\ Magnitude of Proton CurRents Compared TO Electron CURRENTS}

The following is an estimate of how solar proton events (or the inner belt proton environment) can contribute to the total current measured by the SURF instrument within Merlin. From a charging perspective, only the net current is of importance. However, if SURF is employed as an electron monitor, the proton current would be seen as "contamination." Whereas a large SPE has not yet occurred during the Giove-A mission, a small event was recorded on December 13, 2006, associated with a CME.

This problem is approached in two stages; first, the shapes of various SPE spectra are used as inputs to Mulassis [25] to compute what fraction of incident protons are stopped in the three current-measuring plates within SURF. Then, the normalization parameters of the spectra are used to calculate the absolute "positive currents" that will cancel out some or all of the electron "negative currents" that SURF routinely measures.

\section{A. Stage 1: SPE Spectra}

Five SPE spectra are used as Mulassis inputs:

1) SPE September 29, 1989: spectrum derived from GOES and ground neutron monitor data;

2) SPE October 24, 1989: spectrum derived from GOES and ground neutron monitor data;

3) SPE August 4, 1972: spectral shape derived from total fluence Weibull fit and then normalized to a peak $(>10 \mathrm{MeV})$ flux of $10^{6} \mathrm{~cm}^{-2} \mathrm{~s}^{-1}$; 


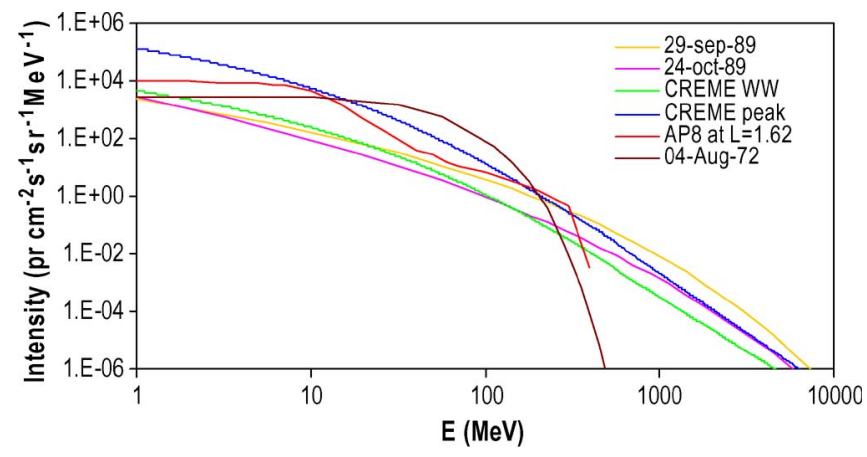

Fig. 13. SPE spectra used as inputs to Mulassis tool. The spectra for September 29, 1989 and October 24, 1989 are derived for the peak time of high energy protons.

\begin{tabular}{|c|c|}
\hline Plate 1 & \\
\hline \multicolumn{2}{|l|}{ Plate 2} \\
\hline & $0.5 \mathrm{~mm}$ \\
\hline Plate 3 & $1 \mathrm{~mm}$ \\
\hline
\end{tabular}

Fig. 14. Schematic diagram of SURF layers in Mulassis. Green areas represent aluminum, and white areas represent a vacuum. The top layer is spacecraft shielding. All layers are $0.5 \mathrm{~mm}$ unless otherwise labeled. Width is arbitrary as Mulassis is a 1-D simulation tool.

4) CREME96 worst week spectrum: 180-h average over series of events in October 1989;

5) CREME96 peak flux spectrum: peak 5-min averaged fluxes observed on GOES in October 1989.

It should be noted that the functional forms for spectra 1) and 2) are derived using empirical data at $>100 \mathrm{MeV}$ and then extrapolated to lower energies. As it is the protons in the energy range $10-100 \mathrm{MeV}$ that are responsible for most of the positive current in the plates, this extrapolation may not accurately represent the relevant part of the spectrum for the first two curves in Fig. 13. As stated previously, the August 1972 SPE spectrum is derived by normalizing the total fluence model of Xapsos to a (Spenvis) quoted peak integral $(>10 \mathrm{MeV})$ flux of $10^{6}$ protons $\mathrm{cm}^{-2} \mathrm{~s}^{-1}$. This corresponds to an equivalent event duration (at peak flux) of $5 \mathrm{~h}$. The August 1972 event spectrum can also be closely fitted with a simple exponential function with a folding energy of $\sim 30 \mathrm{MeV}$. Hence, although this event is soft in that it contained very few high energy protons, the spectral peak occurs in a regime that coincides with SURF's maximum sensitivity to proton contamination.

The Mulassis simulations were performed with layered geometry as shown in Fig. 14. An isotropic proton source was used in each case.

The fraction of incident protons deposited in each SURF plate was calculated by comparing proton flux tallies at the lower edge of each aluminum layer. The results are given in Table I. The equivalent figures for an exponential electron spectrum peaking at $\sim 1 \mathrm{MeV}$ are approximately $18 \%, 10 \%$, and $9 \%$. The percentages of Van Allen belt protons that become trapped are also given for comparison.
TABLE I

Percentage of Incident SPE $>1$-MeV Protons That Become Trapped IN ONE of the Three Respective Aluminum SURF Plates

\begin{tabular}{lccc}
\hline \hline & Top Plate & Middle Plate & Bottom Plate \\
\hline 29-Sep-89 & 8 & 4 & 4 \\
24-Oct-89 & 6 & 3 & 2 \\
4-Aug-72 & 12 & 8 & 11 \\
CRÈME WW & 7 & 3 & 3 \\
CRÈME peak & 6 & 2 & 2 \\
AP8@ L=1.62 & 12 & 4 & 3 \\
SPE average & 7 & 3 & 3 \\
\hline
\end{tabular}

TABLE II

Proton-Induced SURF Plate CurRents in Femtoamperes Per SQuARE CENTIMETER FOR DIFFERENT SPE SPECTRA

\begin{tabular}{lccc}
\hline \hline & Top plate & Middle plate & Bottom plate \\
\hline 29-Sep-89 & 0.29 & 0.14 & 0.17 \\
24-Oct-89 & 0.14 & 0.07 & 0.06 \\
4-Aug-72 & 6.16 & 4.48 & 5.68 \\
CRĖME WW & 0.40 & 0.18 & 0.15 \\
CRÈME peak & 8.37 & 2.63 & 2.85 \\
AP8 @ L=1.62 & 5.46 & 1.65 & 1.11 \\
\hline
\end{tabular}

\section{B. Stage 2: Positive SURF Plate Currents}

Having established the fraction of SPE protons that are expected to become trapped in the current-measuring SURF plates, it is trivial to calculate the expected proton-induced positive currents that will contaminate the electron current measurements—-see Table II. The equivalent figures for trapped protons at $L=1.62$ are also given for comparison. The values in Table II for September 29, 1989 and October 24, 1989 rely on normalizations derived from the peak in the high energy proton flux. The extrapolated low energy component (responsible for proton-induced currents) may therefore be an underestimate of the peak level contamination observed in SURF.

This demonstrates that the technique of extrapolating SPE spectral functions to lower energies from high energy empirical data does seem to produce results on similar orders of magnitude to other data-i.e., the CREME96 worst week model, which is derived from the same time period as the October 1989 SPE (GOES data are also used to derive this spectrum although possibly inclusive of lower energy bands).

More pertinently, this shows that the intensities of the SPEs are likely to produce only negligible currents within the SURF plates. For example, the measured SURF current in plate 1 during the SPE on December 13, 2006 was approximately $1.2 \mathrm{pA} / \mathrm{cm}^{2}\left(1200 \mathrm{fA} / \mathrm{cm}^{2}\right)$. If this was masking a proton positive current on the same order of magnitude as the September 29, 1989 event, then the true electron current would only be $\sim 0.025 \%$ higher than was measured (in actual fact, the December 2007 event was roughly an order of magnitude less intense in terms of $>10-\mathrm{MeV}$ protons than September 1989; therefore, the effect should be even more negligible). A nominally softer event, such as that witnessed in August 1972, actually represents a more severe worst case scenario than CREME96's peak flux model for two of the three current collecting plates; however, the currents are still low when compared to electron current enhancements during SPEs.

This can be contrasted with trapped radiation models (AE8 and AP8 for electrons and protons, respectively) which, for an $L$ shell value of $\sim 1.6$ (orbit altitude $\sim 4000 \mathrm{~km}$ ), predict flux 


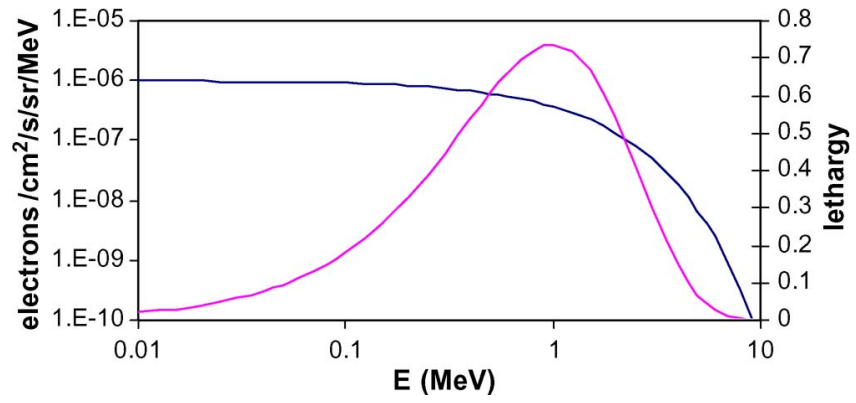

Fig. 15. Template two-parameter electron spectrum. The lethargy spectrum $(E * f(E))$ is also plotted to illustrate the energy ranges in which the majority of flux is contained. The $y$-axis units in both cases are arbitrary and unimportant.

levels what would be expected to induce proton currents in the SURF plates comparable in magnitude to those listed in Table II for the CREME96 peak flux case. These "positive currents" are calculated to be reasonably significant fractions of the negative electron-induced currents at this orbit altitude: $6 \%, 23 \%$, and $23 \%$ for the top, middle, and bottom SURF plates, respectively. These figures are based on equivalent Mulassis simulations using electron input spectra derived from the AE8 model. Actual SURF measurements from the version of the instrument that flew on the STRV1d satellite are up to approximately a factor of two higher $\left(\sim 200 \mathrm{fA} / \mathrm{cm}^{2}\right.$ for the directly comparable top plate); therefore, the proton contamination in this environment may in fact be lower.

\section{APPENDIX B \\ SPECTRAL DETERMINATION}

The basic method is to begin with a parameterized template electron spectrum, multiply this by the known response functions of the three SURF current-measuring plates, and thus determine the predicted plate currents as a function of the initial parameters. Real plate current measurements may then be used to obtain a best fit electron spectrum for the ambient environment.

In the absence of detailed spectral information, the template electron spectrum should be represented by a simple function. Previously, this has taken the form of exponential energy dependence for the integral intensity. The differential form of this function must also follow an exponential; therefore, we have

$$
f(E)=A e^{-\frac{E}{E_{0}}}
$$

where $f(E)$ has units of electrons per square centimeter per second per steradian per megaelectronvolt.

This template spectrum is plotted in Fig. 15 with $E_{o}=$ 1.5 $\mathrm{MeV}$ and an arbitrary value for the proportionality constant $A$.

A crucial part of this process is the accurate knowledge of the response function of the three current-measuring plates contained within SURF. The current measured by each collecting plate essentially represents a count of the environmental electrons that have successfully penetrated the shielding above the plate but have then dissipated all their remaining energy within the plate material and thus become trapped. Therefore, optimum electron energies will correspond to each plate where the energy is sufficient to pass through the shielding layers but insufficient to pass completely through the plate. Monoenergetic isotropic electron sources were used in the DICTAT tool to predict charging currents in each of the three SURF plates; the results are plotted in the main text.

Once the template spectrum $f(E)$ has been defined and the plate response functions $r_{n}(E)$ are known, the overall plate current measurements can be predicted using the following integral:

$$
J_{n}=\int f(E) \cdot r_{n}(E) \cdot d E
$$

which becomes

$$
J_{n}=A \int r_{n}(E) \cdot e^{-\frac{E}{E_{0}}} d E .
$$

If the response functions $\left(r_{n}\right)$ are known in functional form, then the aforementioned integrals may be solved analytically to give plate currents as direct functions of $E_{o}$. In the more realistic case where the response functions are determined numerically, the dependence of plate currents on $E_{o}$ must also be presented numerically.

By calculating two independent plate current ratios (e.g., $\mathrm{J} 1 / \mathrm{J} 2$ and $\mathbf{J} 2 / \mathrm{J} 3$ ), the proportionality constant $(A)$ can be eliminated as a factor leaving two values and two $E_{0}$-dependent variables to be used to determine the spectral hardness. These are

$$
\left(\frac{J_{1}}{J_{2}}\right)_{m} \quad\left(\frac{J_{2}}{J_{3}}\right)_{m} \frac{J_{1}}{J_{2}}\left(E_{0}\right) \quad \frac{J_{2}}{J_{3}}\left(E_{0}\right)
$$

which are plate 1/plate 2 current ratio (measured), plate 2/plate 3 current ratio (measured), plate 1/plate 2 current ratio (as a function of $E_{0}$ ), and plate 2/plate 3 current ratio (as a function of $\left.E_{0}\right)$, respectively.

By systematically varying $E_{0}$, the best fit-to-data value may be determined by minimizing an appropriate statistical measure of the deviation of the model from the data. If empirical errors are well known for the measured plate current values, then a Chi-squared minimization technique is appropriate. In the absence of such errors, an alternative may be used, equivalent to least squares fitting in simple linear problems. As ratios are involved, it is appropriate to define the statistic in logarithmic terms, for example, let the minimization statistic $S$ be defined as

$$
S=\sqrt{\left[\ln \left(\frac{J_{1}}{J_{2}}\right)_{m}-\ln \left(\frac{J_{1}}{J_{2}}\left(E_{0}\right)\right)\right]^{2}+\left[\ln \left(\frac{J_{2}}{J_{3}}\right)_{m}-\ln \left(\frac{J_{2}}{J_{3}}\left(E_{0}\right)\right)\right]^{2}} .
$$

$S$ then uses the logarithmic difference between model prediction and measured value for two plate current ratios, combined in quadrature. Finding the value of $E_{0}$ that yields the lowest possible value of $S$ is a viable method for determining the spectral hardness of the actual electron environment. Having determined the best fit to $E_{0}$, the three predicted plate currents will yield three different values of the proportionality constant $A$. A simple average can then be taken to find the second parameter of the template spectrum. 


\section{ACKNOWLEDGMENT}

The authors would like to thank the whole Giove-A team for creating a successful mission from which these data are gathered.

\section{REFERENCES}

[1] D. N. Baker, X. Li, N. Turner, J. B. Blake et al., "Recurrent geomagnetic storms and relativistic electron enhancements in the outer magnetosphere: ISTP coordinated measurements," J. Geophys. Res., vol. 102, no. A7, pp. 14 141-14 148, 1986.

[2] G. D. Reeves, R. H. W. Friedel, D. D. Belian, M. M. Meier et al., "The relativistic electron response at geosynchronous orbit during the January 1997 magnetic storm," J. Geophys. Res., vol. 103, no. A8, pp. $17559-17570,1998$.

[3] M. D. Violet and A. R. Fredrickson, "Spacecraft anomalies on the CRRES satellite correlated with the environment and insulator samples," IEEE Trans. Nucl. Sci., vol. 40, no. 6, pp. 1512-1520, Dec. 1993.

[4] G. L. Wrenn, D. J. Rodgers, and K. A. Ryden, "A solar cycle of spacecraft anomalies due to internal charging," Ann. Geophys., vol. 20, pp. 953-956, 2002.

[5] A. R. Fredrickson, "Upsets related to spacecraft charging," IEEE Trans. Nucl. Sci., vol. 43, no. 2, pp. 426-441, Apr. 1996.

[6] Avoiding Problems Caused by Spacecraft On-Orbit Internal Charging Effects, Feb. 1999. NASA-HDBK-4002.

[7] D. J. Rodgers and K. A. Ryden, "Internal charging in space," presented at the Proc. 7th Spacecraft Charging Technology Conf., Norrdwijk, The Netherlands, 2001, ESA-SP476.

[8] F. P. Wenaas, M. J. Treadaway, T. M. Flanagan, C. E. Mallon, and R. Denson, "High energy electron induced discharges in printed circuit boards," IEEE Trans. Nucl. Sci., vol. NS-26, no. 6, pp. 5152-5155, Dec. 1979.

[9] A. R. Fredrickson, E. G. Holeman, and E. G. Mullen, "Characteristics of spontaneous electrical discharging of various insulators in space irradiation," IEEE Trans. Nucl. Sci., vol. 39, no. 6, pp. 1773-1982, Dec. 1992.

[10] K. A. Ryden, P. A. Morris, D. J. Rodgers, R. M. Bielby, P. R. Knight, and J. Sørensen, "Anomalies in digital electronics under realistic space electron conditions," in Proc. 7th RADECS Conf., Noordwijk, The Netherlands, Sep. 2003, pp. 593-597.

[11] B. Taylor, C. I. Underwood, H. D. R. Evans, K. A. Ryden, D. Rodgers, E. J. Daly, G. Mandorlo, M. Falcone, P. A. Morris, and R. G. Prieto, "Results from the Galileo Giove-A radiation monitors and comparison with existing radiation belt models," IEEE Trans. Nucl. Sci., vol. 54, no. 4, pp. 1076-1081, Aug. 2007.

[12] K. A. Ryden, H. Jolly, A. Frydland, and P. Morris, "A compact electrostatic charging monitor for spacecraft," in Proc. 5th RADECS Conf., Loire, France, Sep. 1999, pp. 120-125. Abbaye-de-Fontevraud.

[13] K. A. Ryden, D. J. Rodgers, P. A. Morris, A. D. Frydland, H. S. Jolly, and C. S. Dyer, "Direct measurement of internal charging currents in geostationary transfer orbit," in Proc. 6th RADECS Conf., Grenoble, France, Sep. 10-14, 2001, pp. 44-50.

[14] J. Sorensen, D. J. Rodgers, K. A. Ryden, P. M. Latham, G. L. Wrenn, L. Levy, and G. Panabiere, "ESA's tools for internal charging," IEEE Trans. Nucl. Sci., vol. 47, no. 3, pp. 491-497, Jun. 2000.

[15] [Online]. Available: www.spenvis.oma.be

[16] K. A. Ryden, P. A. Morris, D. J. Rodgers, R. Bielby, P. R. Knight, and J. Sorensen, "Improved demonstration of internal charging hazards using the 'Realistic Electron Environment Facility (REEF)'," in Proc. 8th Spacecraft Charging Technol. Conf., Huntsville, AL, Oct. 20-24, 2003. [CD-ROM]

[17] N. A. Tsyganenko, "Global quantitative models of the geomagnetic field in the cislunar magnetosphere for different disturbance levels," Planet. Space Sci., vol. 35, pp. 1347-1358, 1987.

[18] [Online]. Available: http://www.dxlc.com/solar/coronal_holes.html

[19] [Online]. Available: http://cdaw.gsfc.nasa.gov/cme_list/
[20] ESA Mission Controllers React to Solar Flare. [Online]. Available: http://www.esa.int/esaCP/SEMB49QJNVE_index_0.html

[21] G. L. Wrenn, D. J. Rodgers, and P. Buehler, "Modeling the outer belt enhancements of penetrating electrons," J. Spacecr. Rockets, vol. 37, pp. 408-415, 2000

[22] D. J. Rodgers, K. A. Hunter, and G. L. Wrenn, "The FLUMIC electron environment model," in Proc. 8th Spacecraft Charging Technol. Conf., Huntsville, AL, Oct. 2004. NASA/CP-2004-213091.

[23] J. L. Vette, The AE-8 Trapped Electron Model Environment, 1991 NSSDC/WDC-A-R\&S 91-24.

[24] [Online]. Available: www.swpc.noaa.gov/rt_plots/elec_3d.html

[25] F. Lei, P. R. Truscott, C. S. Dyer, B. Quaghebeur, D. Heynderickx, P. Nieminen, H. Evans, and E. Daly, "MULASSIS: A Geant4-based multilayered shielding simulation tool," IEEE Trans. Nucl. Sci, vol. 49, no. 6 , pp. 2788-2793, Dec. 2002.

Keith A. Ryden (M'08), photograph and biography not available at the time of publication.

Paul A. Morris, photograph and biography not available at the time of publication.

Karen A. Ford, photograph and biography not available at the time of publication.

Alexander D. P. Hands, photograph and biography not available at the time of publication.

Clive S. Dyer (M'04-SM'07), photograph and biography not available at the time of publication.

Ben Taylor (M'06), photograph and biography not available at the time of publication.

Craig I. Underwood (M'03), photograph and biography not available at the time of publication.

David J. Rodgers, photograph and biography not available at the time of publication.

Giuseppe Mandorlo, photograph and biography not available at the time of publication.

Giuliano Gatti, photograph and biography not available at the time of publication.

Hugh D. R. Evans, photograph and biography not available at the time of publication.

Eamonn J. Daly, photograph and biography not available at the time of publication. 